\title{
PRÁCTICAS CULTURALES VINCULADAS AL CUIDADO DE LA SALUD Y PERCEPCIÓN SOBRE LA ATENCIÓN EN ESTABLECIMIENTOS DE SALUD EN RESIDENTES DE CENTROS POBLADOS ALTO-ANDINOS DE HUANCAVELICA, PERÚ
}

\author{
Félix Valenzuela-Oré ${ }^{1, a}$, Franco Romaní-Romaní2,b, Betty M. Monteza-Facho ${ }^{1, c}$, Duilio Fuentes-Delgado ${ }^{3, \mathrm{~d}}$, \\ Enma Vilchez-Buitron ${ }^{4, e}$, Oswaldo Salaverry-García ${ }^{5, f}$
}

\begin{abstract}
RESUMEN
Las desigualdades en el acceso y la calidad de atención en salud persisten, especialmente en poblaciones andinas, como la colectividad Chopcca. Describimos la percepción sobre la atención en establecimientos de salud y las prácticas vinculadas al cuidado de la salud en residentes de centros poblados de la región Huancavelica. Realizamos un estudio transversal mediante cuestionario estructurado en 775 residentes de centros poblados urbanos y rurales de los distritos de Yauli y Paucará. 68,7\% refieren usar siempre plantas medicinales para curarse, $86,7 \%$ nunca realizó pago a la tierra, $81,4 \%$ no ha practicado el coca qaway o sara qaway. $88,5 \%$ acude al establecimiento de salud cuando enferma. $57,4 \%$ refiere que el personal de salud siempre brinda confianza y seguridad, dicha proporción es mayor en zonas rurales que urbanas $(64,6 \%$ versus $28,8 \%)$. Las comunidades estudiadas demandan los servicios de salud ofrecidos por el sistema formal. Sin embargo, persisten prácticas culturales tradicionales, siendo la más importante el uso de plantas medicinales.
\end{abstract}

Palabras clave: Percepción; Conocimientos; Actitudes y Práctica en Salud; Atención primaria de salud; Medicina tradicional; Andes; Perú (fuente: DeCS BIREME).

\section{CULTURAL PRACTICES LINKED TO HEALTH CARE AND PERCEPTION ON THE ATTENTION HEALTH FACILITIES IN RESIDENTS OF HIGH- ANDEAN SETTLEMENTS IN HUANCAVELICA, PERU}

\begin{abstract}
Inequalities in terms of access and quality of healthcare persist, especially among Andean populations, such as the community of Chopcca. Here, we describe the perception of healthcare provided in medical facilities as well as the practices linked to health care in residents of settlements in the Huancavelica region. We carried out a cross-sectional study by means of a structured questionnaire administered to 775 residents of urban and rural towns of the Yauli and Paucará districts. $68.7 \%$ refer always using medicinal plants to cure themselves; $86.7 \%$ never made a "payment" to Mother Earth; $81.4 \%$ has not practiced coca qaway or sara qaway. $88.5 \%$ of respondents visit the medical facility when they fall ill. $57.4 \%$ refer that the healthcare staff always transmits confidence and security, this proportion is higher in rural vs. urban areas $(64.6 \%$ versus $28.8 \%$ ). The communities under study demand the healthcare services offered by the formal system; nevertheless, traditional cultural practices persist, being the most important one the use of medicinal plants.
\end{abstract}

Keywords: Perception; Health knowledge; Attitudes; Practice; Primary health care; Traditional medicine; Andean; Peru (source: MeSH NLM).

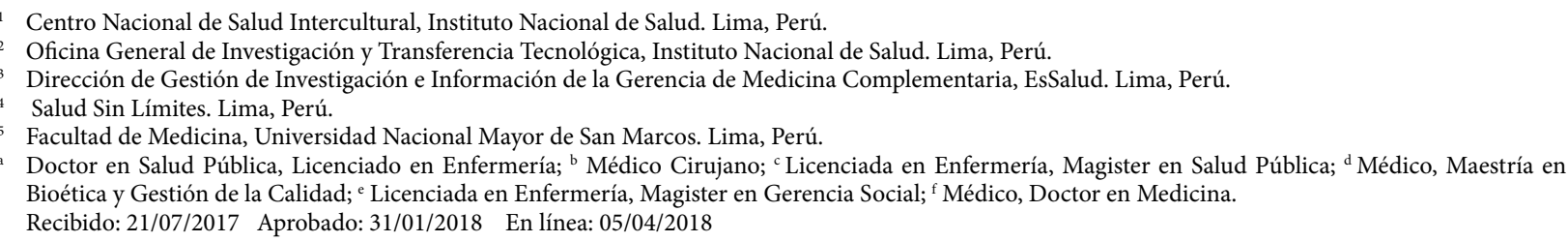

Citar como: Valenzuela-Oré F, Romaní-Romaní F, Monteza-Facho BM, Fuentes-Delgado D, Vilchez-Buitron E, Salaverry-García O. Prácticas culturales vinculadas al cuidado de la salud y percepción sobre la atención en establecimientos de salud en residentes de centros poblados alto-andinos de Huancavelica, Perú. Rev Peru Med Exp Salud Publica. 2018;35(1):84-92. doi: 10.17843/rpmesp.2018.351.3603. 


\section{INTRODUCCIÓN}

La reforma de salud en el Perú tiene como objetivos fundamentales el aseguramiento universal en salud (AUS), la descentralización en salud y el fortalecimiento del primer nivel de atención (1), los cuales deben cumplirse considerando la diversidad cultural y étnica del país, tal como busca la política sectorial de salud intercultural (2). Sin embargo, se observa que la reforma tiene avances aún limitados, y persisten desigualdades significativas en el acceso y el estado de salud entre diferentes segmentos socioeconómicos ${ }^{(3)}$; particularmente en poblaciones indígenas $\mathrm{u}$ originarias, tanto amazónicas como andinas ${ }^{(4-6)}$.

Según el Ministerio de Cultura, la diversidad cultural del país se plasma oficialmente en 55 poblaciones indígenas u originarias ${ }^{(7)}$, las cuales se encuentran entre los sectores sociales de mayor exclusión y desventaja ${ }^{(8,9)}$. Cada uno de estos pueblos originarios tuvo un desarrollo diferenciado, generando instituciones culturales propias como el uso de lenguas ancestrales y una cosmovisión particular, de la cual deriva un sistema propio de cuidados de la salud. Diversos estudios han descrito barreras derivadas de la percepción sobre los servicios de salud, así como de las prácticas culturales, que afectan la implementación de los servicios brindados por el sistema de salud formal ${ }^{(10-12)}$.

Para el 2016, en grupo vulnerables del Perú (aquellos con lengua materna quechua, aymara u otra lengua nativa), la proporción de personas que acudieron a un establecimiento de salud en caso de enfermedad, síntoma o molestia fue del $66 \%$ entre varones, y del $61 \%$ en mujeres, con mínimas diferencias entre el ámbito urbano y rural. Los distritos de Yauli y Paucará de la región Huancavelica comprenden grupos vulnerables. Estos son comunidades campesinas de la colectividad Chopcca, los cuales cuentan con agentes tradicionales de salud entre ellos 'curanderos', 'hampiq (médicos andinos), parteros, $\mathrm{u}$ otros que brindan atención ante problemas de salud ${ }^{(13)}$. El objetivo del estudio fue describir las prácticas culturales vinculadas al cuidado de la salud y su percepción sobre la atención en establecimientos de salud de centros poblados alto-andinos de la región Huancavelica.

\section{EL ESTUDIO}

La región Huancavelica se encuentra en la zona sur andina del Perú a 450 kilómetros de Lima (capital). Presenta indicadores sanitarios decrecientes en los últimos años, por ejemplo, la cobertura de vacunación BGC muestra una disminución de $68,3 \%$ (2009) a 51,6\% (2013), la lactancia materna exclusiva (LME) en menores de seis meses pasó de $60 \%(2009)$ a $48,3 \%(2013)$, la cobertura de parejas protegidas por métodos anticonceptivos pasó de $54,4 \%$ (2009) a 40,5\% (2013); entre otros ${ }^{(14)}$.

\section{MENSAJES CLAVE}

Motivación para realizarelestudio. Laatención en losestablecimientos de salud en el Perú, tiene muchas dificultades, persisten desigualdades en el acceso a servicios de calidad, existen prácticas culturales singulares por lo que es necesario describir percepciones y diseñar estrategias que permitan mejorar.

Principales hallazgos. En general se observa un patrón de percepción favorable con respecto a la atención de salud, aún persisten las prácticas de medicina tradicional, uso de plantas medicinales, síndromes y enfermedades culturales, y búsqueda de atención con agentes tradicionales.

Implicancias. Es necesario abordar la coexistencia entre la medicina tradicional y la oferta de servicios en establecimientos de salud, diseñando estrategias que permitan ofrecer un servicio con pertinencia cultural.

Se realizó un estudio transversal en comunidades alto andinas de los distritos de Yauli y Paucará, ubicados en las provincias de Huancavelica y Acobamba, respectivamente. Estos distritos fueron seleccionados pues en sus territorios se encuentran asentadas las comunidades de la colectividad Chopcca (13). El distrito de Yauli tiene una población estimada (año 2015) de 16526 habitantes y una proporción de pobreza del $87 \%$, el distrito de Paucará cuenta con 36713 habitantes (2015) y 89,1\% de pobreza.

La población de estudio fueron residentes de comunidades (centros poblados urbanos y rurales) de los distritos seleccionados. El distrito de Yauli está conformado por siete centros poblados urbanos y 27 rurales, mientras Paucará tiene nueve centros poblados urbanos y 11 rurales. Los centros poblados fueron considerados como conglomerados y unidades primarias de muestreo, las unidades secundarias de muestreo fueron las viviendas. Se considera como centro poblado urbano, a aquel que tiene como mínimo 100 viviendas agrupadas contiguamente formando manzanas y calles. El centro poblado rural es aquel que no tiene más de 100 viviendas contiguas formando manzanas ni calles, ni es capital de distrito; o que, teniendo más de 100 viviendas, estas se encuentran semidispersas o totalmente dispersas.

Se estimó un tamaño muestral para la estimación de una proporción, se consideró como tamaño poblacional el número total de viviendas para ambos distritos (Yauli $=4823$, Paucará $=3873$, total=8 696 ); la proporción esperada fue del $50 \%$, un error relativo del $10 \%$ y un efecto de diseño de 2. Con dichos supuestos el tamaño muestral fue de 736 viviendas. Se realizó un muestreo de conglomerados sin sub muestreo, mediante la selección sistemática de conglomerados con probabilidad proporcional al número de viviendas. De esta forma se aseguró la selección de conglomerados procedentes del distrito de Yauli y Paucará, y que sean centros poblados urbanos y rurales. Fueron 
seleccionados tres conglomerados en el distrito de Yauli (el centro poblado urbano "Centro" y los centros poblados rurales "San Juan de Ccarhuacc" $y$ "Chucllaccasa") y dos en Paucará (el centro poblado urbano "Pampacruz" y el centro poblado rural "Mejorada"). Los criterios de inclusión para las unidades de análisis fueron: tener $\geq 18$ años, ser el jefe del hogar o representante al momento de la visita, residir en la comunidad por más de un año y brindar consentimiento informado por escrito.

Se aplicó un cuestionario estructurado que recogió datos demográficos, reporte de prácticas culturales (idioma, acude a urqu qayay, práctica de pago a la tierra, práctica del coca qaway/sara qaway, uso de plantas medicinales, conocimientos de males, práctica de curaciones, atención con curanderos/parteras, participación en fiestas comunitarias), características del uso y acceso a establecimientos de salud (EE. SS.) (lugar de atención, frecuencia de uso de EE. SS., tiempo de traslado a EE. SS., tiempo de espera para atención, tipo de atención, idioma del personal del EE. SS, entendimiento del personal), percepción sobre la atención del personal de salud en el consultorio (confianza y seguridad, pregunta sobre motivo de consulta, privacidad, explicación de procedimientos, preguntas sobre contexto familiar, comunicación pausada, oportunidad de preguntar, resolución del problema de salud, interés sobre formas de curación tradicional, tiempo para atención), percepción sobre estrategias empleadas por el EE. SS. (participación en la programación de actividades de salud comunitaria, promoción de atención de salud y los programas sociales), percepción sobre labor en promoción y prevención en salud (realización de charlas de prevención de enfermedades, encuentros de madres/ gestantes, campañas/ferias de salud).

El instrumento fue adaptado de la Encuesta de Satisfacción de Usuarios Externos de Servicios de Salud del Ministerio de Salud, posteriormente fue sometido a juicio de tres expertos en salud intercultural para evaluar la validez de contenido y finalmente a prueba piloto en una comunidad del distrito de Acoria (centro poblado "Los Ángeles de Ccarahuasa") para mejorar la viabilidad del instrumento, afinando el tiempo empleado por sujeto de estudio, la claridad de las preguntas y su llenado.

La investigación fue presentada a las autoridades del gobierno regional, dirección de salud de Huancavelica, municipalidades distritales y dirigentes de las comunidades seleccionadas. Se identificó a personal de salud local que demostraron dominio del quechua y experiencia previa en captura de información en campo, posteriormente fueron entrenados en aspectos de interculturalidad y uso de herramientas como diálogo intercultural en salud. Dicho personal aplicó el instrumento de recolección de datos. Previamente con el apoyo de las autoridades locales se construyó el mapeo de viviendas de las comunidades seleccionadas. Las visitas fueron realizadas casa por casa en noviembre de 2014, invitándose a participar a aquellas unidades de análisis que cumplieran los criterios de inclusión. La aplicación del cuestionario tuvo una duración promedio de 30 minutos. La información fue ingresada a una base de datos en el programa Excel MS, el ingreso de los datos fue realizado por dos digitadores ciegos e independientes, posteriormente el investigador principal realizó el control de calidad de la totalidad de datos ingresados.

Se realizó el análisis descriptivo mediante frecuencias y porcentajes de las características demográficas para el total de la muestra y estratificado por tipo de centro poblado (urbano y rural). Se realizó el análisis descriptivo de las prácticas culturales, y la percepción de los usuarios sobre los servicios de salud según tipo de centro poblado, se compararon proporciones para muestras independientes mediante la prueba chi cuadrado. Se consideró un valor $p<0,05$ como significativo. El análisis estadístico fue realizado en el paquete SPSS versión 22 para Windows (Chicago, IL, USA).

El estudio fue aprobado por el Comité de Ética en Investigación del Instituto Nacional de Salud. La dirección regional de salud Huancavelica autorizó la ejecución del estudio. Se solicitó consentimiento informado por escrito a los sujetos elegibles, y se garantizó que el tratamiento y almacenamiento de datos se realice en forma anónima.

\section{HALLAZGOS}

Se invitó a participar a 775 personas, la totalidad aceptó participar y completó el cuestionario. De la muestra enrolada, $401(51,7 \%)$ fueron residentes del centro poblado rural "San Juan de Ccarhuacc", 175 (22,6\%) del centro poblado rural "Chucllaccasa", $113(14,6 \%)$ del centro poblado urbano "Pampacruz", 43 (5,5\%) del centro poblado rural "Mejorada" y $43(5,5 \%)$ del centro poblado urbano "Centro". El 79,9\% ( $n=619)$ reside en centros poblados rurales.

$495(63,9 \%)$ fueron mujeres, $625(80,6 \%)$ refieren ser residentes permanentes de sus comunidades, $507(65,4 \%)$ refieren hablar sólo quechua. La media y desviación estándar (DE) para el número de hijos en las familias de centros poblados urbanos fue de $3,35 \pm 2,17$, mientras que en centros poblados rurales fue de $3,72 \pm 2,57(p=0,02)$. $90,3 \%$ refieren tener ingresos mensuales $<500$ soles/mes, siendo esta proporción mayor en centros poblados rurales $(96,9 \%)$ (Tabla 1).

El urqu qayaq (curandero que invoca a los cerros para indagar las causas de una dolencia) nunca ha sido practicado por el $85,5 \%$ de los participantes, el pago a la tierra nunca fue realizada por el $86,7 \%$ de participantes, la práctica del coca qaway o sara qaway (lectura de la hoja de 
Tabla 1. Características sociodemográficas de personas encuestadas según tipo de centro poblado en los distritos de Yauli y Paucará, región Huancavelica, 2014

\begin{tabular}{|c|c|c|c|}
\hline \multirow{2}{*}{ Características } & \multicolumn{2}{|c|}{ Centro poblado } & \multirow[b]{2}{*}{$\begin{array}{l}\text { Total (775) } \\
\mathrm{n}(\%)\end{array}$} \\
\hline & $\begin{array}{c}\text { Urbano (156) } \\
\text { n (\%) }\end{array}$ & $\begin{array}{l}\text { Rural (619) } \\
\text { n (\%) }\end{array}$ & \\
\hline \multicolumn{4}{|l|}{ Grupo etario (años) } \\
\hline $18-30$ & $48(30,8)$ & $247(39,9)$ & $295(38,1)$ \\
\hline $31-40$ & $32(20,5)$ & $157(25,4)$ & $189(24,4)$ \\
\hline $41-50$ & $27(17,3)$ & $91(14,7)$ & $118(15,2)$ \\
\hline $51-60$ & $30(19,2)$ & $73(11,8)$ & $103(13,3)$ \\
\hline$>60$ & $19(12,2)$ & $51(8,2)$ & $70(9,0)$ \\
\hline \multicolumn{4}{|l|}{ Sexo } \\
\hline Femenino & $124(79,5)$ & $371(59,9)$ & $495(63,9)$ \\
\hline Masculino & $32(20,5)$ & $248(40,1)$ & $280(36,1)$ \\
\hline \multicolumn{4}{|l|}{ Nivel de instrucción } \\
\hline Sin instrucción & $47(30,1)$ & $147(23,7)$ & $194(25,0)$ \\
\hline Primaria & $52(33,3)$ & $252(40,7)$ & $304(39,2)$ \\
\hline Secundaria & $51(32,7)$ & $217(35,1)$ & $268(34,6)$ \\
\hline Superior & $6(3,8)$ & $3(0,5)$ & $9(1,2)$ \\
\hline \multicolumn{4}{|l|}{ Ingreso mensual (soles) } \\
\hline$<500$ & $100(64,1)$ & $600(96,9)$ & $700(90,3)$ \\
\hline$\geq 500$ & $56(35,9)$ & $19(3,1)$ & $75(9,7)$ \\
\hline \multicolumn{4}{|l|}{ Idioma } \\
\hline Quechua & $89(57,1)$ & $418(67,5)$ & $507(65,4)$ \\
\hline Castellano & $17(10,9)$ & $2(0,3)$ & $19(2,5)$ \\
\hline Quechua y Castellano & $50(32,1)$ & $199(32,1)$ & $249(32,1)$ \\
\hline \multicolumn{4}{|l|}{ Religión } \\
\hline Católico & $104(66,7)$ & $439(70,9)$ & $543(70,1)$ \\
\hline Evangélico & $50(32,1)$ & $170(27,5)$ & $220(28,4)$ \\
\hline Otro & 0 & $3(0,5)$ & $3(0,4)$ \\
\hline Ninguno & $2(1,3)$ & $7(1,1)$ & $9(1,2)$ \\
\hline \multicolumn{4}{|l|}{ Tiempo de residencia } \\
\hline Desde nacimiento & $94(60,3)$ & $531(85,8)$ & $625(80,6)$ \\
\hline Reside entre $1-3$ años & $20(12,8)$ & $21(3,4)$ & $41(5,3)$ \\
\hline Reside entre 4 - 6 años & $6(3,8)$ & $9(1,5)$ & $15(1,9)$ \\
\hline $\begin{array}{l}\text { Reside más de } 6 \text { años pero no } \\
\text { desde nacimiento }\end{array}$ & $36(23,1)$ & $58(9,4)$ & $94(12,1)$ \\
\hline \multicolumn{4}{|l|}{ Seguro de salud } \\
\hline Sí & $154(98,7)$ & $617(99,7)$ & $771(99,5)$ \\
\hline No & $2(1,3)$ & $2(0,3)$ & $4(0,5)$ \\
\hline \multicolumn{4}{|l|}{ Número de hijos por familia } \\
\hline Ninguno & $7(4,5)$ & $7(1,1)$ & $14(1,8)$ \\
\hline 1 a 2 & $62(39,7)$ & $248(40,1)$ & $310(40,0)$ \\
\hline 3 a 4 & $42(26,9)$ & $155(25,0)$ & $197(25,4)$ \\
\hline$\geq 5$ & $45(28,8)$ & $209(33,8)$ & $254(32,8)$ \\
\hline
\end{tabular}


coca y grano de maíz para averiguar causas de dolencias, respectivamente) nunca fue realizada por el $81,4 \%$. El reporte de usar siempre plantas medicinales fue en el $68,7 \%$. En centros poblados rurales, el $10,4 \%$ refiere nunca haber usado plantas medicinales mientras en centros poblados urbanos dicho porcentaje fue $2,6 \%$ (Tabla 2).

$88,5 \%$ de participantes refiere buscar atención en EE. SS., esta proporción es mayor en centros poblados urbanos $(95,5 \%$ versus $86,8 \%, p=0,007)$. En el ámbito rural, el uso de medicina tradicional es mayor $(5,7 \%$ versus $3,8 \%)$. Respecto a la accesibilidad, en centros poblados urbanos, el $85,9 \%$ refiere llegar al EE. SS. en menos de 30 minutos, mientras que en áreas rurales dicha proporción es de $60,3 \%$. En centros poblados urbanos, el $51,3 \%$ refiere acudir al EE. SS. por atención pediátrica, en el ámbito rural principalmente buscan atención materno-infantil (55,3\%). El $60,1 \%$ refiere que el personal que lo atiende habla quechua y español, dicho porcentaje es mayor en el área rural $(62,8 \%)$ (Tabla 3$)$.

Tabla 2. Prácticas culturales vinculadas al cuidado de la salud en residentes de cinco centros poblados rurales y urbanos de Huancavelica, Perú 2014

\begin{tabular}{|c|c|c|c|c|}
\hline \multirow[b]{2}{*}{ Características } & \multicolumn{2}{|c|}{ Tipo de centro poblado } & \multirow[b]{2}{*}{$\begin{array}{l}\text { Total } \\
\text { n (\%) }\end{array}$} & \multirow[b]{2}{*}{ Valor de $p$} \\
\hline & $\begin{array}{l}\text { Urbano } \\
\text { n (\%) }\end{array}$ & $\begin{array}{l}\text { Rural } \\
\text { n (\%) }\end{array}$ & & \\
\hline \multicolumn{5}{|c|}{ ¿Acude a urqu qayaq cuando tiene problemas de salud? } \\
\hline Siempre & $5(3,2)$ & $31(5,0)$ & $36(4,7)$ & 0,259 \\
\hline A veces & $20(12,8)$ & $56(9,1)$ & $76(9,8)$ & \\
\hline Nunca & $131(84,0)$ & $529(85,9)$ & $660(85,5)$ & \\
\hline \multicolumn{5}{|c|}{ ¿Realizan pago a la tierra? } \\
\hline Siempre & $5(3,2)$ & $32(5,2)$ & $37(4,8)$ & $<0,01$ \\
\hline A veces & $27(17,3)$ & $39(6,3)$ & $66(8,5)$ & \\
\hline Nunca & $124(79,5)$ & $548(88,5)$ & $672(86,7)$ & \\
\hline \multicolumn{5}{|c|}{ ¿Practican el coca qaway o el sara qaway? } \\
\hline Siempre & $8(5,1)$ & $52(8,4)$ & $60(7,7)$ & 0,390 \\
\hline A veces & $17(10,9)$ & $67(10,8)$ & $84(10,8)$ & \\
\hline Nunca & $131(84,0)$ & $500(64,5)$ & $631(81,4)$ & \\
\hline \multicolumn{5}{|c|}{ ¿Usa plantas medicinales para curarse? } \\
\hline Siempre & $131(84,0)$ & $401(64,9)$ & $532(68,7)$ & $<0,01$ \\
\hline A veces & $21(13,5)$ & $153(24,8)$ & $174(22,5)$ & \\
\hline Nunca & $4(2,6)$ & $64(10,4)$ & $68(8,8)$ & \\
\hline \multicolumn{5}{|c|}{$\begin{array}{l}\text { ¿Creen en males de salud como el sillki, ñati, urihua entre } \\
\text { otros? }\end{array}$} \\
\hline Siempre & $54(34,6)$ & $166(26,8)$ & $220(28,4)$ & $<0,01$ \\
\hline A veces & $68(43,6)$ & $218(35,2)$ & $286(36,9)$ & \\
\hline Nunca & $34(21,8)$ & $235(38,0)$ & $269(34,7)$ & \\
\hline \multicolumn{5}{|c|}{$\begin{array}{l}\text { ¿Practica algún tipo de curación?: qayapu (llamar, suplicar al } \\
\text { espíritu en caso de susto), pasada con huevo, soba con cuy? }\end{array}$} \\
\hline Siempre & $48(30,8)$ & $64(10,3)$ & $112(14,5)$ & $<0,01$ \\
\hline A veces & $59(37,8)$ & $114(18,4)$ & $173(22,3)$ & \\
\hline Nunca & $49(31,4)$ & $441(71,2)$ & $490(63,2)$ & \\
\hline \multicolumn{5}{|c|}{ ¿Acuden al curandero o partera frente a problemas de salud? } \\
\hline Siempre & $8(5,1)$ & $25(4,0)$ & $33(4,3)$ & 0,821 \\
\hline A veces & $19(12,2)$ & $73(11,8)$ & $92(11,9)$ & \\
\hline Nunca & $129(82,7)$ & $521(84,2)$ & $650(83,9)$ & \\
\hline \multicolumn{5}{|c|}{ ¿Participan en fiestas costumbristas? } \\
\hline Siempre & $22(14,1)$ & $266(43,0)$ & $288(37,2)$ & $<0,01$ \\
\hline A veces & $47(30,1)$ & $217(35,1)$ & $264(34,1)$ & \\
\hline Nunca & $87(55,8)$ & $136(22,0)$ & $223(28,8)$ & \\
\hline
\end{tabular}


Tabla 3. Características de uso y acceso a los establecimientos de salud en residentes de cinco centros poblados rurales y urbanos de Huancavelica, Perú 2014

\begin{tabular}{|c|c|c|c|c|}
\hline \multirow[b]{2}{*}{ Características } & \multicolumn{2}{|c|}{ Tipo de centro poblado } & \multirow[b]{2}{*}{$\begin{array}{l}\text { Total } \\
\text { n (\%) }\end{array}$} & \multirow[b]{2}{*}{$\begin{array}{l}\text { Valor } \\
\text { de } p\end{array}$} \\
\hline & $\begin{array}{c}\text { Urbano } \\
\text { n (\%) }\end{array}$ & $\begin{array}{l}\text { Rural } \\
\text { n (\%) }\end{array}$ & & \\
\hline \multicolumn{5}{|l|}{ ¿Dónde acude cuando se enferma? } \\
\hline Usa medicina tradicional para curarse & $6(3,8)$ & $35(5,7)$ & $41(5,3)$ & $<0,01$ \\
\hline Se auto medica con productos farmacéuticos & $1(0,6)$ & $8(1,3)$ & $9(1,2)$ & \\
\hline Se atiende con el curandero & $0(0)$ & $39(6,3)$ & $39(5,0)$ & \\
\hline Asiste al establecimiento de salud & $149(95,5)$ & $537(86,8)$ & $686(88,5)$ & \\
\hline \multicolumn{5}{|c|}{$\begin{array}{l}\text { En el último año ¿cuántas veces acudió al establecimiento de } \\
\text { salud? }\end{array}$} \\
\hline Sólo una vez & $18(11,7)$ & $51(8,3)$ & $69(8,9)$ & $<0,01$ \\
\hline De dos a tres veces & $65(42,2)$ & $186(30,1)$ & $251(32,5)$ & \\
\hline De cuatro a cinco & $31(20,1)$ & $81(13,1)$ & $112(14,5)$ & \\
\hline Más de cinco & $40(26,0)$ & $300(48,5)$ & $340(44,0)$ & \\
\hline \multicolumn{5}{|c|}{$\begin{array}{l}\text { ¿Cuánto tiempo le toma llegar al establecimiento de salud? } \\
\text { (minutos) }\end{array}$} \\
\hline$<30$ & $134(85,9)$ & $373(60,3)$ & $507(65,4)$ & $<0,01$ \\
\hline 30 a 60 & $13(8,3)$ & $157(25,4)$ & $170(21,9)$ & \\
\hline 61 a 120 & $3(1,9)$ & $64(10,3)$ & $67(8,6)$ & \\
\hline$>120$ & $6(3,8)$ & $25(4,0)$ & $31(4,0)$ & \\
\hline \multicolumn{5}{|c|}{$\begin{array}{l}\text { ¿Cuánto tiempo espera para ser atendido en el establecimiento } \\
\text { de salud? (minutos) }\end{array}$} \\
\hline$<30$ & $13(8,3)$ & $178(28,8)$ & $191(24,6)$ & 0,01 \\
\hline 30 a 60 & $53(34,0)$ & $355(57,4)$ & $408(52,6)$ & \\
\hline 61 a 120 & $45(28,8)$ & $69(11,1)$ & $114(14,7)$ & \\
\hline$>120$ & $45(28,8)$ & $17(2,7)$ & $62(8,0)$ & \\
\hline \multicolumn{5}{|c|}{$\begin{array}{l}\text { ¿Qué tipo de atención buscan generalmente en el establecimiento } \\
\text { de salud? }\end{array}$} \\
\hline Atención para niño & $80(51,3)$ & $121(19,5)$ & $201(25,9)$ & $<0,01$ \\
\hline Atención materno-infantil & $18(11,5)$ & $342(55,3)$ & $360(46,5)$ & \\
\hline Atención para adulto & $10(6,4)$ & $99(16,0)$ & $109(14,1)$ & \\
\hline Atención para adulto mayor & $32(20,5)$ & $36(5,8)$ & $68(8,8)$ & \\
\hline Urgencias y emergencias & $16(10,3)$ & $21(3,4)$ & $37(4,8)$ & \\
\hline \multicolumn{5}{|l|}{ ¿Qué idioma habla el personal que lo atiende? } \\
\hline Sólo quechua & $13(8,3)$ & $46(7,4)$ & $59(7,6)$ & $<0,01$ \\
\hline Sólo castellano & $66(42,3)$ & $184(29,7)$ & $250(32,3)$ & \\
\hline Quechua y castellano & $77(49,4)$ & $389(62,8)$ & $466(60,1)$ & \\
\hline \multicolumn{5}{|l|}{ ¿Entiende las explicaciones del personal de salud? } \\
\hline Sí, entiendo todo & $92(59,0)$ & $276(44,6)$ & $368(47,5)$ & 0,01 \\
\hline Sí, entiendo algunas cosas & $59(37,8)$ & $321(51,9)$ & $380(49,0)$ & \\
\hline No entiendo nada & $5(3,2)$ & $22(3,6)$ & $27(3,5)$ & \\
\hline
\end{tabular}

$57,4 \%$ refieren que el personal de salud muestra siempre confianzayseguridad. Encentrospobladosurbanosunmayor porcentaje $(39,7 \%)$ refiere la ausencia de esta característica comparado con centros poblados rurales $(5,2 \%)$. En el ámbito rural, $71,2 \%$ refieren que el personal de salud siempre pregunta el motivo de consulta, dicha proporción es menor en el ámbito urbano (49,4\%). Igualmente, a nivel rural una mayor proporción de participantes refieren que siempre se explica los procedimientos durante la consulta $(70,3 \%)$ y pregunta sobre el contexto familiar $(70,1 \%)$. El $4,6 \%$ de participantes refiere que el personal de salud no le resuelve el problema motivo de consulta (Tabla 4). 
Tabla 4. Percepciones de residentes de centros poblados rurales y urbanos sobre la atención de salud en consultorio y las actividades de promoción y prevención de salud implementadas por los establecimientos de salud, Huancavelica, Perú 2014

\begin{tabular}{|c|c|c|c|c|}
\hline \multirow[b]{2}{*}{ Características } & \multicolumn{2}{|c|}{ Tipo de centro poblado } & \multirow[b]{2}{*}{$\begin{array}{l}\text { Total } \\
\text { n (\%) }\end{array}$} & \multirow[b]{2}{*}{$\begin{array}{l}\text { Valor } \\
\text { de p }\end{array}$} \\
\hline & $\begin{array}{c}\text { Urbano } \\
\text { n (\%) }\end{array}$ & $\begin{array}{l}\text { Rural } \\
\text { n (\%) }\end{array}$ & & \\
\hline \multicolumn{5}{|c|}{ Atención en consultorio } \\
\hline \multicolumn{5}{|c|}{ El personal de salud muestra confianza y seguridad } \\
\hline Siempre & $45(28,8)$ & $400(64,6)$ & $445(57,4)$ & $<0,01$ \\
\hline Casi siempre & $49(31,4)$ & $187(30,2)$ & $236(30,5)$ & \\
\hline Nunca & $62(39,7)$ & $32(5,2)$ & $94(12,1)$ & \\
\hline \multicolumn{5}{|c|}{ El personal de salud pregunta el motivo de consulta } \\
\hline Siempre & $77(49,4)$ & $441(71,2)$ & $518(66,8)$ & $<0,01$ \\
\hline Casi siempre & $68(43,6)$ & $146(23,6)$ & $214(27,6)$ & \\
\hline Nunca & $11(7,1)$ & $32(5,2)$ & $43(5,5)$ & \\
\hline \multicolumn{5}{|c|}{ El personal asegura la privacidad } \\
\hline Siempre & $113(72,4)$ & $482(77,9)$ & $595(76,8)$ & 0,339 \\
\hline Casi siempre & $41(26,3)$ & $129(20,8)$ & $170(21,9)$ & \\
\hline Nunca & $2(1,3)$ & $8(1,3)$ & $10(1,3)$ & \\
\hline \multicolumn{5}{|c|}{ El personal de salud examina explicando los procedimientos } \\
\hline Siempre & $78(50,0)$ & $435(70,3)$ & $513(66,2)$ & $<0,01$ \\
\hline Casi siempre & $66(42,3)$ & $156(25,2)$ & $222(28,6)$ & \\
\hline Nunca & $12(7,7)$ & $28(4,5)$ & $40(5,2)$ & \\
\hline \multicolumn{5}{|c|}{ El personal de salud pregunta sobre el contexto familiar } \\
\hline Siempre & $41(26,3)$ & $434(70,1)$ & $475(61,3)$ & $<0,01$ \\
\hline Casi siempre & $36(23,1)$ & $124(20,0)$ & $160(20,6)$ & \\
\hline Nunca & $79(50,6)$ & $61(9,9)$ & $140(18,1)$ & \\
\hline \multicolumn{5}{|c|}{ El personal de salud le habla pausado } \\
\hline Siempre & $94(60,3)$ & $361(58,3)$ & $455(58,7)$ & 0,336 \\
\hline Casi siempre & $53(34,0)$ & $236(38,1)$ & $289(37,3)$ & \\
\hline Nunca & $9(5,8)$ & $22(3,6)$ & $31(4,0)$ & \\
\hline \multicolumn{5}{|c|}{ El personal brinda oportunidad para preguntar } \\
\hline Siempre & $66(42,3)$ & $353(57,0)$ & $419(54,1)$ & $<0,01$ \\
\hline Casi siempre & $77(49,4)$ & $202(32,6)$ & $279(36,0)$ & \\
\hline Nunca & $13(8,3)$ & $64(10,3)$ & $77(9,9)$ & \\
\hline \multicolumn{5}{|c|}{ El personal resuelve el problema de salud } \\
\hline Siempre & $53(34,0)$ & $363(58,6)$ & $416(53,7)$ & $<0,01$ \\
\hline Casi siempre & $94(60,3)$ & $229(37,0)$ & $323(41,7)$ & \\
\hline Nunca & $9(5,8)$ & $27(4,4)$ & $36(4,6)$ & \\
\hline \multicolumn{5}{|c|}{$\begin{array}{l}\text { El personal de salud permite que el paciente explique cómo se cura } \\
\text { tradicionalmente }\end{array}$} \\
\hline Siempre & $39(25,0)$ & $228(36,8)$ & $267(34,5)$ & $<0,01$ \\
\hline Casi siempre & $78(50,0)$ & $287(46,4)$ & $365(47,1)$ & \\
\hline Nunca & $39(25,0)$ & $104(16,8)$ & $143(18,5)$ & \\
\hline \multicolumn{5}{|c|}{ El personal brinda tiempo suficiente al usuario } \\
\hline Siempre & $95(60,9)$ & $390(63,0)$ & $485(62,6)$ & 0,678 \\
\hline Casi siempre & $52(33,3)$ & $203(32,8)$ & $255(32,9)$ & \\
\hline Nunca & $9(5,8)$ & $26(4,2)$ & $35(4,5)$ & \\
\hline \multicolumn{5}{|c|}{ Actividades de promoción y prevención en salud } \\
\hline \multicolumn{5}{|c|}{ El establecimiento de salud brinda charlas sobre prevención de enfermedades } \\
\hline Siempre & $38(24,4)$ & $398(64,3)$ & $436(56,3)$ & $<0,01$ \\
\hline Casi siempre & $80(51,3)$ & $149(24,1)$ & $229(29,5)$ & \\
\hline Nunca & $35(22,4)$ & $37(6,0)$ & $72(9,3)$ & \\
\hline No opina & $3(1,9)$ & $3(5,7)$ & $38(4,9)$ & \\
\hline El establecimiento & & & & \\
\hline Siempre & $24(15,4)$ & $210(33,9)$ & $234(30,2)$ & $<0,01$ \\
\hline Casi siempre & $86(55,1)$ & $297(48,0)$ & $383(49,4)$ & \\
\hline Nunca & $31(19,9)$ & $59(9,5)$ & $90(11,6)$ & \\
\hline No opina & $15(9,6)$ & $53(8,6)$ & $68(8,8)$ & \\
\hline El establecimiento & & & & \\
\hline Siempre & $16(10,3)$ & $147(23,7)$ & $163(21,0)$ & $<0,01$ \\
\hline Casi siempre & $61(39,1)$ & $293(47,3)$ & $354(45,7)$ & \\
\hline Nunca & $72(46,2)$ & $124(20,0)$ & $196(25,3)$ & \\
\hline No opina & $7(4,5)$ & $55(8,9)$ & $62(8,0)$ & \\
\hline
\end{tabular}


$43,9 \%$ refieren que el EE. SS. nunca incluye a los usuarios en su programación de actividades. El 17,8\% $(n=110)$ de participantes de centros poblados rurales refieren que el $\mathrm{EE}$. SS. siempre los incluye en la programación, y $40,7 \%(n=252)$ algunas veces; en el ámbito urbano dichas frecuencias son menores, $7,7 \%(n=12)$ para siempre y $39,1 \%(n=61)$ para algunas veces $(p<0,01)$. El $77,4 \%$ refiere que los programas sociales (“Juntos", "Pensión 65 ") siempre condicionan la búsqueda de atención en los EE. SS., en el ámbito rural dicha proporción es del $76,7 \%(n=475)$ y en el urbano de $80 \%(n=124)$. El 9,3\% de la muestra refiere que el $E E$. SS. no brinda charlas sobre prevención de enfermedades, dicha proporción es significativamente mayor en el ámbito urbano $(22,4 \%)$ comparado con el rural $(6,0 \%)(p<0,01)$. El $11,6 \%$ reporta que los EE. SS. no organizan encuentros de madres/gestantes, siendo menos frecuente su realización en el ámbito urbano, tendencia similar se observa para la organización de campañas/ferias de salud (Tabla 4).

\section{DISCUSIÓN}

El $90 \%$ de participantes refiere acudir a los EE. SS. cuando enferman, este indicador es especialmente importante en comunidades con familias que reportan ingresos menores a 500 soles y mayoritariamente quechua hablantes, y donde previamente, en el 2007 el Instituto Nacional de Estadística e Informática reportó que en centros poblados como "Mejorada" y "Chucllaccasa" el aseguramiento al Seguro Integral de Salud era del $48,4 \%$ y $56 \%$, respectivamente ${ }^{(13)}$.

Respecto a prácticas culturales vinculadas al cuidado de la salud, el acudir al urqu qayaq, el pago a la tierra, y el coca qaway o sara qaway no son frecuentes en los distritos estudiados. El uso de plantas medicinales es la práctica más frecuente, especialmente en los centros poblados urbanos, con proporciones similares a las encontradas en usuarios de dos centros hospitalarios de la ciudad de Cusco ${ }^{(15)}$. La frecuencia de uso estaría asociado a la variada y abundante oferta de plantas medicinales en los mercados ${ }^{(16,17)}$. Se ha reportado que el uso de la medicina tradicional en población Chopcca ha disminuido, para el 2008 era común que la mayoría de los integrantes de esta colectividad acuda a los yerberos y use plantas medicinales antes que atenderse en los EE. SS. Las causas atribuidas para decidir por la medicina tradicional eran la ineficiencia de los medicamentos prescritos y la presencia de personal técnico en sustitución de médicos ${ }^{(13)}$.

Nuestros resultados muestran que persisten prácticas culturales vinculadas con el diagnóstico y tratamiento de enfermedades, sin embargo, estas no son tan frecuentes como en investigaciones previas. Esto podría tener múltiples causas: en nuestra muestra la religión predominante es el cristianismo, la cual se le atribuye la censura del uso de la medicina tradicional (13); por estrategias asociadas al otorgamiento de programas sociales ("Juntos", "Pensión
65 ") que condicionan al uso de los servicios de salud; por sanciones económicas para situaciones de parto domiciliario ${ }^{(18)}$; y por el incremento de la cobertura del aseguramiento en salud.

A pesar de la mejora cuantitativa en la cobertura prestacional del sector formal de salud, aún persisten, en diferentes grados prácticas de medicina tradicional, como el uso de síndromes y enfermedades culturales, práctica de curaciones y búsqueda de atención con agentes tradicionales. Dicha coexistencia conforma un ecosistema mixto de oferta de atención en salud, el cual ha sido descrito en comunidades andinas ${ }^{(5)}$ y amazónicas del Perú (4)

Respecto a las características del acceso a los EE. SS. los participantes de centros poblados rurales refieren un mayor tiempo de llegada al establecimiento, un $14 \%$ de usuarios en zonas rurales refieren más de una hora para llegar al EE. SS. Sin embargo, se observó que el tiempo de espera para ser atendido tiende a ser menor comparado con los EE. SS en zonas urbanas. Las dificultades en el acceso se deberían a la falta de carreteras, unidades de transporte motorizado, y la irregular geografía del ámbito rural ${ }^{(13)}$.

Otra característica que fomentaría el uso de los servicios de salud, especialmente en las zonas rurales, sería el idioma que maneja el personal de salud (quechua) y el nivel de entendimiento de los mensajes ofrecidos. Dichas características estarían asociadas con el número de visitas al EE. SS. Casi la mitad de participantes de los centros poblados rurales refiere acudir al EE. SS. más de cinco veces por año, en zonas urbanas dicha proporción es menor.

Se observa un patrón de percepciones más favorables con respecto a la atención de salud en los centros poblados rurales, especialmente en la confianza y seguridad demostrada por el personal de salud, en la indagación del contexto familiar, y la forma de comunicación. Una mayor proporción de participantes de las zonas rurales refiere que el personal de salud siempre explica los procedimientos que realiza, siempre da oportunidad de preguntar al paciente, y permite al paciente explicar como este se cura con medicina tradicional. Esta favorable percepción del usuario estaría asociada con la percepción de resolución del problema de salud, la cual es mayor en zonas rurales. Dichos hallazgos son congruentes con lo reportado por la Encuesta Nacional de Satisfacción de usuarios de salud del 2014, en la cual el $65,2 \%$ de usuarios de la región Huancavelica refiere sentirse satisfecho con el servicio, y sólo $10 \%$ refiere sentirse insatisfecho ${ }^{(14)}$.

El presente estudio tuvo las siguientes limitaciones: la generalización de los resultados está limitada a las comunidades estudiadas, por otro lado, el sesgo de auto- 
reporte generado por deseabilidad social pudo emerger en la medición de la percepción respecto a la atención en los EE. SS. El estudio por tener un enfoque cuantitativo no pudo explorar aspectos culturales que expliquen el acceso y uso a los servicios de salud, y su coexistencia con prácticas culturales tradicionales.

En conclusión, los residentes de centros poblados alto andinos rurales perciben de mejor manera la atención que reciben en los EE. SS. lo cual podría conllevar a que preferentemente busquen cuidado de la salud en el sistema formal; sin embargo, algunas prácticas culturales persisten, especialmente el uso de plantas medicinales. El mayor uso de servicios de salud propiciado por una serie de estrategias del sector público, ha tenido como efecto secundario el abandono progresivo de prácticas tradicionales vinculadas al cuidado de la salud (especialmente en el ámbito rural), área donde la medicina tradicional de la colectividad Chopcca fue históricamente difundida.

Contribución de los autores: FVO y OSG participaron en la concepción y diseño del estudio. FVO, BMF y EVB realizaron la recolección de la información. FRR y FVO realizaron el análisis e interpretación de los resultados y la redacción del artículo. Todos los autores participaron en la revisión crítica y aprobación final del manuscrito.

Fuentes de financiamiento: Instituto Nacional de Salud.

Conflictos de interés: Los autores declaran no tener conflictos de interés.

\section{REFERENCIAS BIBLIOGRÁFICAS}

1. Velásquez A, Suarez D, Nepo-Linares E. Reforma del sector salud en el Perú: derecho, gobernanza, cobertura universal y respuesta contra riesgos sanitarios. Rev Peru Med Exp Salud Pública. 2016;33(3):546-55.

2. Aprueban la Política Sectorial de Salud Intercultural. Decreto Supremo $\mathrm{N}^{\circ} 016$ 2016-SA. 2016.

3. Perú. Encuesta Demográfica y de Salud Familiar - ENDES. 2014 (Nacional y Departamental). Instituto Nacional de Estadística e Informática. 2015.

4. Williamson J, Ramirez R, Wingfield T. Health, healthcare access, and use of traditional versus modern medicine in remote Peruvian Amazon communities: a descriptive study of knowledge, attitudes, and practices. Am J Trop Med Hyg. 2015;92(4):857-64.

5. Mathez-Stiefel S-L, Vandebroek I, Rist S. Can Andean medicine coexist with biomedical healthcare? A comparison of two rural communities in Peru and Bolivia. J Ethnobiol Ethnomedicine. 2012; 8:26.

6. Brierley CK, Suarez N, Arora G, Graham D. Healthcare access and health beliefs of the indigenous peoples in remote Amazonian Peru. Am J Trop Med Hyg. 2014; 90(1):180-3.

7. Base de Datos de Pueblos Indígenas u Originarios. Lima: Ministerio de Cultura;
[Internet]. Available from: http://bdpi. cultura.gob.pe/lista-de-pueblos-indigenas

8. Dirección General de Epidemiología. Ministerio de Salud. Análisis de la Situación de Salud del Pueblo Aymara, Puno, 2010. Lima: Ministerio de Salud; 2010.

9. Dirección General de Epidemiología. Ministerio de Salud. Análisis de Situación de Salud del Pueblo Nanti del Alto Camisea. Lima: Ministerio de Salud; 2014.

10. Aparco JP, Huaman L. Barreras y facilitadores a la suplementación con micronutrientes en polvo. Percepciones maternas y dinámica de los servicios de salud. Rev Peru Med Exp Salud Publica. 2017;34(4):590-600.

11. Mayca-Pérez J, Medina-Ibañez A, Velásquez-Hurtado JE, Llanos-Zavalaga LF. Representaciones sociales relacionadas a la anemia en niños menores de tres años en comunidades Awajun y Wampis, Perú. Rev Peru Med Exp Salud Pública. 2017; 34(3):414-22.

12. Huamán B, Gushiken A, Benites C, Quiroz F, García L. Prevención de la transmisión materno-infantil del VIH en gestantes y madres awajún y wampis de la región Amazonas en Perú. Rev Peru Med Exp Salud Publica. 2017;34(4):627-32.

13. Pedro R, Marleni M. Los chopcca de Huancavelica. Etnicidad y cultura en el Perú contemporáneo. Colección: Pueblos y tradicional. Lima: Ministerio de Cultura; 2013.
14. Dirección Regional de Salud Huancavelica. Gobierno Regional de Salud Huancavelica. Análisis de Situación de Salud, Región Huancavélica. Huancavelica: Dirección Regional; 2015.

15. Oblitas G, Hernández-Córdova G, Chiclla A, Antich-Barrientos M, Ccorihuamán-Cusitito L, Romaní F. Use of medicinal plants among people attending two reference hospitals in Cuzco, Peru. Rev Peru Med Exp Salud Publica. 2013;30(1):64-8.

16. Bussmann RW, Sharon D. Traditional medicinal plant use in Loja province, Southern Ecuador. J Ethnobiol Ethnomedicine. 2006; 2:44.

17. de la Cruz MG, Malpartida SB, Santiago HB, Jullian V, Bourdy G. Hot and cold: medicinal plant uses in Quechua speaking communities in thehigh Andes (Callejón de Huaylas, Ancash, Perú).J Ethnopharmacol. 2014; 155(2):1093-117.

18. Félix VO, Oswaldo SG, Betty MF, Duilio FD, Omar TV, Enma VB, et al. Percepción del usuario sobre los servicios de salud de la comunidad de Chopcca, Huancavelica, desde una mirada intercultural (Resultado Preliminar). Bol - Inst Nac Salud. 2015; 21(1-2):16-23.

Correspondencia: Félix Valenzuela Oré

Dirección: Jr. General Córdova 1021 Dto. 301. Jesús María, Lima

Correo electrónico:Fvalenzuelao_12@yahoo.es 